\title{
Article \\ A Molecularly Imprinted Polymer Based SPR Sensor for 2-Furaldehyde Determination in Oil Matrices
}

\author{
Maria Pesavento ${ }^{1, *}$, Nunzio Cennamo ${ }^{2} \mathbb{D}$, Luigi Zeni ${ }^{2}\left(\mathbb{D}\right.$ and Letizia De Maria ${ }^{3}(\mathbb{D}$ \\ 1 Department of Chemistry, University of Pavia, Via Taramelli 12, 27100 Pavia, Italy \\ 2 Department of Engineering, University of Campania L. Vanvitelli, Via Roma 29, 81031 Aversa, Italy; \\ nunzio.cennamo@unicampania.it (N.C.); luigi.zeni@unicampania.it (L.Z.) \\ 3 RSE SpA, Via Rubattino 54, 20134 Milan, Italy; Letizia.DeMaria@rse.web.it \\ * Correspondence: maria.pesavento@unipv.it
}

check for updates

Citation: Pesavento, M.; Cennamo,

N.; Zeni, L.; De Maria, L. A

Molecularly Imprinted Polymer Based SPR Sensor for 2-Furaldehyde Determination in Oil Matrices. Appl. Sci. 2021, 11, 10390. https://doi.org/ 10.3390/app112110390

Academic Editor: Andrei Sarbu

Received: 16 October 2021

Accepted: 1 November 2021

Published: 5 November 2021

Publisher's Note: MDPI stays neutral with regard to jurisdictional claims in published maps and institutional affiliations.

Copyright: (c) 2021 by the authors. Licensee MDPI, Basel, Switzerland. This article is an open access article distributed under the terms and conditions of the Creative Commons Attribution (CC BY) license (https:/ / creativecommons.org/licenses/by/ $4.0 /)$.
Featured Application: The work shows the development of an optical chemosensor based on a synthetic receptor, a molecularly imprinted polymer, for a furanic compound, 2-furaldehyde, with a good possibility of application in liquid samples, even very complex, and with high refractive index as a vegetable oil. This opens the way for the wide application of this sensing technique on site and in matrices of high industrial interest.

Abstract: Optical chemosensors with surface plasmon resonance (SPR) transduction are widely employed, even in complex environments, such as those outside the laboratory. In this context, not only the chemical nature but also the physical form of the receptor layer is particularly relevant. Synthetic receptors as molecularly imprinted polymers (MIPs) are well suited. This is demonstrated here in the case of an SPR sensor platform based on a multimode plastic optical fiber, which is very promising for on site application due to the low dimensions and low cost. A specific MIP was used as the receptor, with high affinity for the substance to be determined, 2-furaldehyde, in water. Here, a medium of high refractive index, i.e., vegetable oil, was considered because of the high interest for its determination in industrial diagnostics. The effects of the MIP layer thickness and the washing extent on the quality of the analytical signal were investigated. Better spectra were generated at the thinner MIP layer, while a lower detection limit is reached with extended washing.

Keywords: molecularly imprinted polymer; optical sensor; plastic optical fiber; surface plasmon resonance; 2-furaldehyde; vegetable oil

\section{Introduction}

The need for low-cost and easy-to-use sensing systems is constantly increasing in different application areas, in particular for health, food and environmental controls and rapid screening of contaminants. A wide interest for sensing systems is also present in several industrial contexts, for example for early warnings of failures, with the aim of reducing maintenance costs, or as effective data sources for big data applications and predictive analysis [1,2].

This work focuses on the application of a sensor for the selective detection of furfural, 2-furaldehyde (2-FAL), in vegetable oil. The 2-FAL detection in this kind of matrix is becoming interesting for diagnostic purposes, since it is recognized that the presence of 2-FAL in the oil of oil-insulated electrical equipment is an important marker of the degradation of the insulating system. 2-FAL can be present as a byproduct of the thermal degradation of cellulose, so 2-FAL detection in insulating oil is a crucial task for preventing accelerated aging phenomena of oil-paper insulation (paper windings, pressboards) in transformers [3-5]. These phenomena heavily influence the effective lifetime of power transformers.

Traditional monitoring techniques are typically based on laboratory analyses, mainly chromatographic methods, of representative field-collected samples; this necessitates 
considerable time, effort and expense, and can produce some changes in the sample composition before analysis. An alternative approach consists of portable monitoring systems relying on sensing methods. They appear to be well suited to complement standard analytical methods and, also, can be permanently installed at monitoring sites and can transmit data remotely.

Considering the wide range of application areas, a huge number of bio- and chemoreceptor-based sensors have been described in the last twenty years, among which those based on optical transduction, and in particular on optical fibers, have been shown as well suited for numerous applications [6-11]. The sensing platform used here for the determination of 2-FAL in vegetable oil is based on an optical transduction method, surface plasmon resonance (SPR), on optical fiber, and based on a synthetic chemical receptor, a molecularly imprinted polymer (MIP). It has been previously used for a number of applications in aqueous matrices [12-14], but a different matrix was also considered, i.e., a mineral oil of the isolated transformers [15]. The optical fiber sensing platforms based on SPR allow marker-free detection, and plastic optical fibers (POFs) are especially advantageous for low-cost sensing systems due to their excellent flexibility, easy manipulation, great numerical aperture, large diameter and the fact that plastic can withstand smaller bend radii than glass [16].

The platform proposed here consists of a planar surface of an exposed POF core, embedded in a resin block (D-shaped POF platform), covered with a dielectric layer of photoresist with high RI and a thin gold layer [17]. The receptor layer is deposited on the gold surface. The flat shape of the sensing part is particularly suitable for measurements in a drop, for which no expensive and bulky flow-through cell is required.

MIPs are a class of artificial solid receptors [18-20] containing sites functionally and dimensionally complementary to the target molecular structure, similar to the receptor sites in bioreceptors. The process of MIP preparation is based on a template-assisted synthesis [19]. MIPs often possess recognition properties analogous to natural receptors but have the stability, ease of preparation, micromachining, integrability and low cost of production typical of synthetic materials. Moreover, MIPs can be easily produced as layers in tight contact with the transducing surfaces $[12,14]$. In this form, MIPs are different from the usual bioreceptors, for example, antibodies or aptamers, since the thickness of the layer, while being not larger than a few hundred nanometers, is certainly much higher than that of the bioreceptors layers, usually constituted by only one or a few molecular layers. This is a favorable aspect for sensing applications when considering real matrices with high refractive index (RI).

In the D-shaped POF-SPR platform proposed here, an MIP layer can be easily deposited by a drop coating and spinning procedure, as previously described in several cases [12-14]. In this study, the effect of different factors on the sensor response to 2-FAL when used in a matrix with high RI is investigated, considering as a proof of principle a vegetable insulating oil as the liquid medium, with an RI as high as 1.47. In particular, the effect of the thickness of the MIP layer was examined. The idea is that measurements in a medium with high RI, i.e., higher than those detectable by the sensing platform and the experimental setup proposed here, can still be carried out, taking advantage of the fact that the MIP layer may have an RI lower than that of the considered vegetable oil and that its thickness can be modulated in a simple way. Depending on the thickness of the MIP, the plasmonic wave can penetrate only the MIP layer, or it can reach the fluid above. This has been shown in a previous work in the case of aqueous media [14].

\section{Materials and Methods}

\subsection{Chemicals and Instrumentation}

Divinylbenzene [1321-74-0] (DVB), ethylene glycol dimethacrylate [97-90-5] (EGDMA), methacrylic acid [79-41-4] (MAA), 2,2'-azobisisobutyronitrile [78-67-1] (AIBN) and 2furaldehyde [98-01-1] (2-FAL) were obtained from Sigma-Aldrich, Merk (Darmstadt, Germany). MAA and DVB were purified before use to remove stabilizers by fluxing the 
liquid through a $4 \mathrm{~cm}$ column of alumina (Sigma-Aldrich). All other chemicals were of analytical reagent grade. Pure water was obtained by a Milli-Q system (Merck Millipore, Billerica, MA, USA). A stock solution with $11,600 \mathrm{mg} \mathrm{L}^{-1}$ of 2-FAL was prepared by weighing the liquid and dissolving it in $96 \%$ ethanol. The standard solutions were obtained by successive dilution in the considered solvent, i.e., vegetable oil, obtained from the market and containing less than $0.01 \mathrm{mg} \mathrm{L}^{-1}$ of furanic compounds. The measurement apparatus consisted of a halogen lamp (HL-2000-LL, Ocean Optics, Dunedin, FL, USA) and a spectrometer connected to a PC (USB2000+UV-VIS spectrometer, Ocean Optics, Dunedin, FL, USA). The emission wavelength range of the white light source was from $360 \mathrm{~nm}$ to $1700 \mathrm{~nm}$, whereas the detection range of the spectrometer was from $350 \mathrm{~nm}$ to $1023 \mathrm{~nm}$. The transmission spectra were saved and processed by SpectraSuite software (Ocean Optics, Dunedin, FL, USA). An outline of the experimental setup making possible the spectral interrogation is reported in Figure 1a.

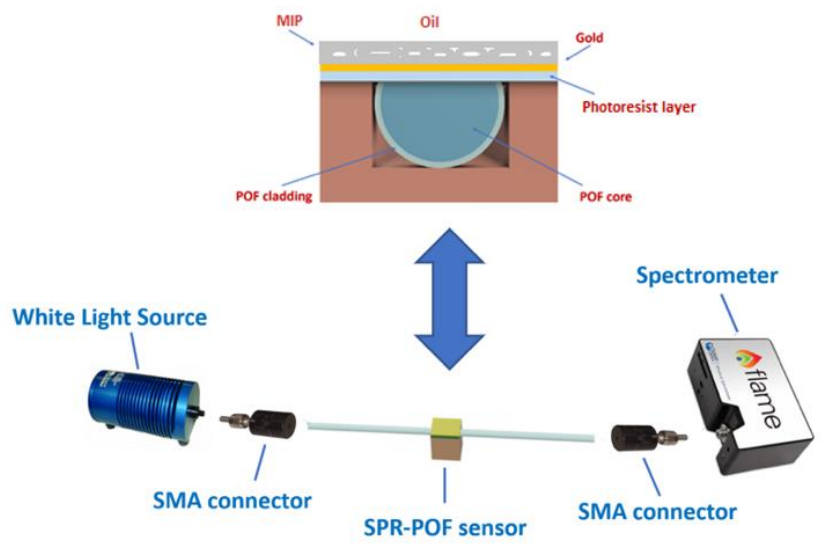

(a)

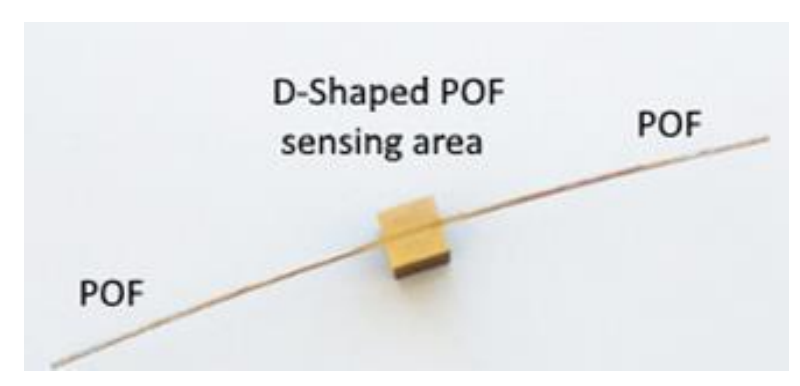

(b)

Figure 1. Optical chemical sensor system. (a) Outline of the experimental setup with a schematic representation of the D-shaped multilayer SPR-POF platform. The brown block represents the resin support $(1 \mathrm{~cm} \times 1 \mathrm{~cm} \times 1 \mathrm{~cm})$ in which the POF (in red) is embedded. The grey layer is the photoresist buffer, and the blue layer is the receptor. SMA is the subminiature version of the connector. The different parts are not in scale. (b) Picture of the SPR-POF platform.

\subsection{Preparation of the D-Shaped Fiber Optic Platform}

The optical platform was based on a multimode POF with a characteristic D-shaped sensing region, obtained as previously reported [12-14]. Briefly, the cladding and partially the core of the POF, held in a specially designed resin support $1 \mathrm{~cm}$ long, were manually erased, producing a flat surface (see Figure 1). One half of the fiber was erased, and a multilayer structure was built up over the exposed core by first depositing a buffer layer (Microposit photoresist, MicroChemCorp., Westborough, MA, USA) of high refractive index with respect to the core, $1.5 \mu \mathrm{m}$ thick, deposited by dropping and spinning at $6000 \mathrm{rpm}$, a thin metal film (gold, $60 \mathrm{~nm}$ thick, deposited by sputtering with an SCD 500, LeicaMicrosystems, Wetzlar, Germany) and, finally, an MIP layer as a specific chemical receptor for 2-FAL detection.

\subsection{Deposition of the Specific MIP Layer}

The prepolymeric mixture was composed of the reagents at molar ratio 1 (2-FAL):4 (MAA):40 (DVB), according to the method previously described [14]. No porogen solvent was used. The cross-linker divinylbenzene (DVB) also acted as the solvent in which the functional monomer (methacrylic acid (MAA)) and the template, 2-FAL, were dissolved. The mixture was uniformly dispersed by sonication and de-aerated with nitrogen for $10 \mathrm{~min}$. Then, the radical initiator AIBN ( $23 \mathrm{mg} / \mathrm{mL}$ of prepolymeric mixture) was added 
to the mixture. An MIP with the same molar composition, but containing ethylene glycol dimethacrylate (EGDMA) instead of DVB, was prepared for comparison purposes.

The MIP layer was prepared by dropping directly over the flat part of the platform a small volume (about $50 \mu \mathrm{L}$ ) of the prepolymeric mixture on the platform maintained in a flat position with the help of the resin support. The prepolymeric mixture expanded spontaneously to cover the erased surface of the POF and of the resin support. The whole structure was spun at a given spin rate, typically at 1000 or $500 \mathrm{rpm}$ for $2 \mathrm{~min}$, and then placed in an oven for $16 \mathrm{~h}$ at $75^{\circ} \mathrm{C}$ for thermal polymerization in air. Finally, the template and oligomeric polymer fragments were removed by repeated washings with $96 \%$ ethanol.

Layers with different thicknesses could be obtained by spinning the prepolymeric mixture at different rates or by depositing multiple layers of MIP. The thickness of the MIP layer is particularly relevant when liquids with high RI are examined.

\subsection{Measurement}

The D-shaped optical platforms embedded in the resin block allowed performing the measurement in a drop simply deposited over the flat surface, $1 \mathrm{~cm} \times 1 \mathrm{~cm}$ wide. The platform was fixed in a miniholder, which was designed to keep the sensing surface in a flat position. A sample drop $(50 \mu \mathrm{L})$ was deposited over the flat part of the sensor, allowed to expand over the sensing surface and the support and equilibrated for $5 \mathrm{~min}$. During this time, the oil drop tended to cover the whole flat surface of the support due to the lipophilic nature of the MIP, which was mainly constituted by DVB or EGDMA.

All the measurements were performed in an air-conditioned room at $25^{\circ} \mathrm{C}$.

The spectra were recorded directly in the drop of the liquid sample positioned over the sensing surface. Depending on the penetration depth of the plasmonic wave, the RI of the dielectric can be influenced by the RI of the liquid, and if this is sufficiently high, the resonance wavelength could even exceed the detectable range. If so, the solvent exchange method [14] must be applied, which is, however, quite complex and time consuming.

The transmission spectra of the SPR-POF-MIP sensors in air, water and oil were normalized to the spectrum of the corresponding bare sensor (SPR-POF-bare) in air (reference spectrum).

\subsection{Characterization of the Optical Sensitivity of the Bare Platform}

Characterization was carried out by measuring the shift of the resonance wavelength in liquid with different refractive indices. These were solutions of glycerin in water at different concentrations, the refractive index of which was measured by an Abbe refractometer [17].

\subsection{Characterization of the Sensor Response in Oil}

Dose-response curves were obtained by plotting the variation of the resonance wavelength in the normalized transmission spectra $(\Delta \lambda)$ in the function of the concentration of 2-FAL. $\Delta \lambda$ refers to the resonance wavelength of a blank solution, i.e., a solution with the same composition of the sample but not containing the analyte of interest.

Considering the limited number of receptor sites in sensors based on MIPs, the response to the concentration of the template can be modeled by an equation derived from the Langmuir adsorption model, which has been successfully applied in previous investigations carried out in aqueous matrices [14]. The relation between the response (DL) and the concentration of the molecule of interest [A] (in mg L $\mathrm{m}^{-1}$ or $\mathrm{M} \mathrm{L}^{-1}$ ), assuming that the signal is directly proportional to the amount of the template in the sensing layer $\left(\Delta \lambda=k \cdot g \cdot c_{\text {Aint }}\right)$, is given by the following equation:

$$
\Delta \lambda=\frac{k g c_{\mathrm{int}} K_{\mathrm{aff}}[\mathrm{A}]}{1+K_{\mathrm{aff}}[\mathrm{A}]}=\frac{\Delta \lambda_{\max } K_{\mathrm{aff}}[\mathrm{A}]}{1+K_{\mathrm{aff}}[\mathrm{A}]}
$$

$K_{\mathrm{aff}}\left(\right.$ in $\mathrm{mg}^{-1} \mathrm{~L}$ or $\mathrm{M}^{-1} \mathrm{~L}$ ) is the affinity constant of the adsorption equilibrium, $c_{\mathrm{int}}$ is the concentration of the specific sites of the MIP (in $\mathrm{mg} \mathrm{g}^{-1}$ ), $\mathrm{g}$ is the polymer mass (in grams) and $\Delta \lambda_{\max }=k \cdot g \cdot c_{\text {int }}$ is the maximum $\Delta \lambda$ at high concentrations of the analyte (i.e., when the analyte saturates all the specific sites). 
If the analyte concentration at the equilibrium in the sample, [A], is equal to the total concentration $\left(c_{\mathrm{A}}\right)$, i.e., when the concentration of the analyte adsorbed is negligible, Equation (1) can be used for evaluating the parameters, in particular $K_{\mathrm{aff}}$, which is useful for characterizing the adsorption equilibria. The second parameter, $\Delta \lambda_{\max }$, depends on the number of sites but also on the sensitivity of the transduction method, which is in principle unknown. Thus, it can only be used for comparative purposes. The parameters of Equation (1) were obtained by Solver, a Microsoft Excel add-on program.

\section{Results}

\subsection{Characterization of SPR-POF-Bare Platforms}

A few D-shaped optical platforms on POF were manually prepared as described (from SPR-POF-A1bare to SPR-POF-A5bare). The spectra in water normalized on the spectra of the same platform in air, registered immediately before the measurement in water, are reported in Figure 2.

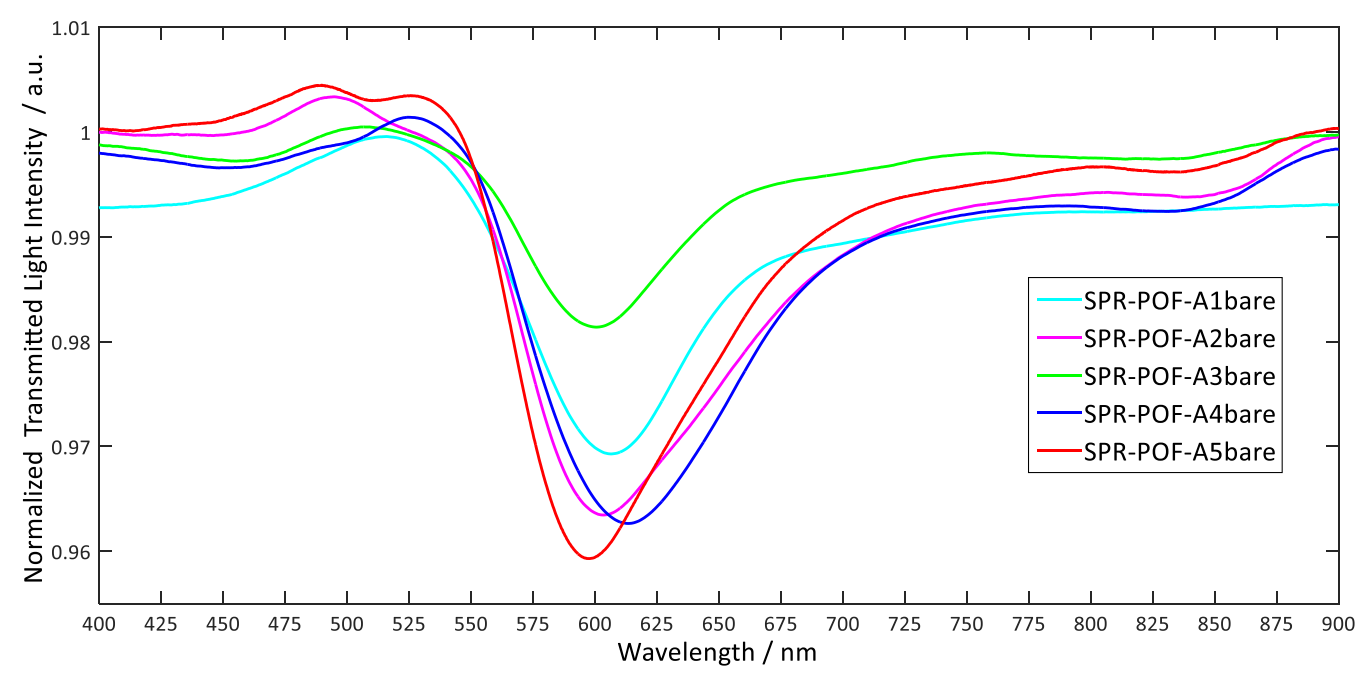

Figure 2. SPR spectra in water of the bare optical platforms, normalized to the spectra in air.

The spectra present some dips between 500 and $510 \mathrm{~nm}$ and at about $600 \mathrm{~nm}$ (average value of $603.5( \pm 2.8) \mathrm{nm})$.

The depths of the dips are different, which could reflect the irreproducibility of the manual preparation. The optical sensitivity was determined for two platforms, SPR-POFA4bare and SPR-POF-A5bare, which show somewhat different spectra in water (see Figure 2). Nevertheless, the sensitivity curves of the two sensors (Figure 3) are similar, showing that the sensitivity is very low below $n=1.33$ and is higher at high RI (about $3500 \mathrm{RIU} / \mathrm{nm}$ ).

\subsection{SPR Spectra of POF-MIP Sensors}

In order to investigate the effect of the thickness of the MIP layer, two kinds of sensors were considered: the first one was prepared on the platform SPR-POF-A5bare by depositing the prepolymeric mixture at $1000 \mathrm{rpm}$ and the second one was prepared by depositing two layers of MIP (SPR-POF-A1MIP2 and SPR-POF-A2MIP2), each one at a spinning rate of $500 \mathrm{rpm}$. They should be covered by a larger amount of MIP than SPR-POF-A5MIP1.

As an example, the spectra in the water of the bare platforms of SPR-POF-A5bare and SPR-POF-A2bare are shown in Figure 4. In both cases, the spectra of the corresponding MIP sensor in air and water, normalized to the spectrum of the bare sensor in air, present several dips, which are sharper and better defined for SPR-POF-A5MIP1. Additionally, the difference between the spectrum in air and that in water is higher in the case of SPR-POF-A5MIP1, which confirms that a lower amount of MIP is deposited. 


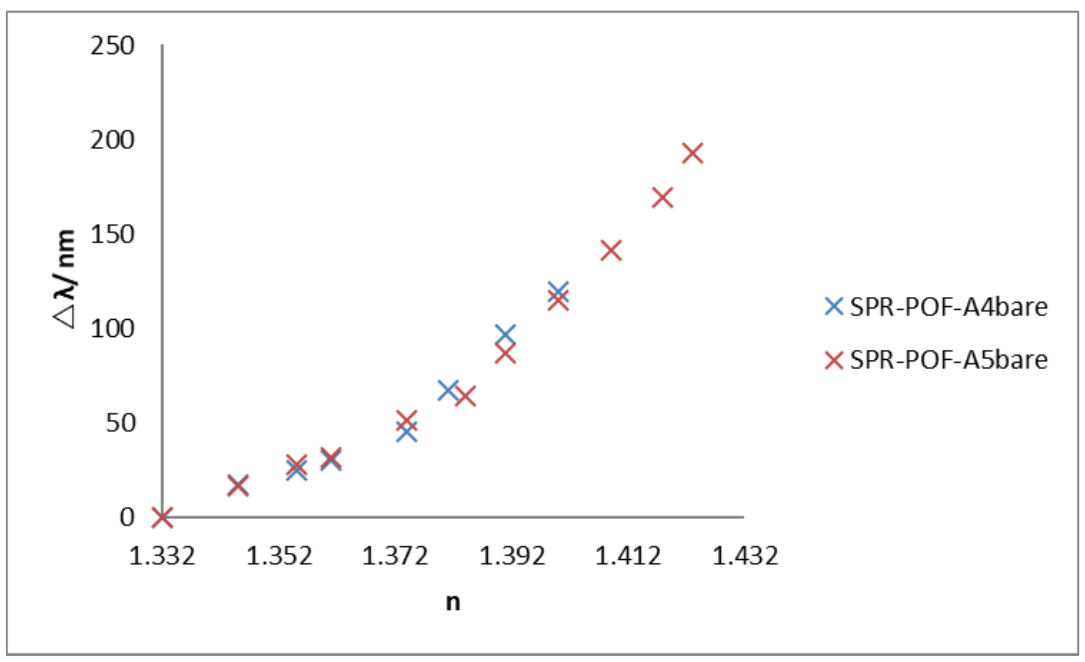

Figure 3. Sensitivity curves of SPR-POF-A4bare and SPR-POF-A5bare in water-glycerin solutions at different RIs.

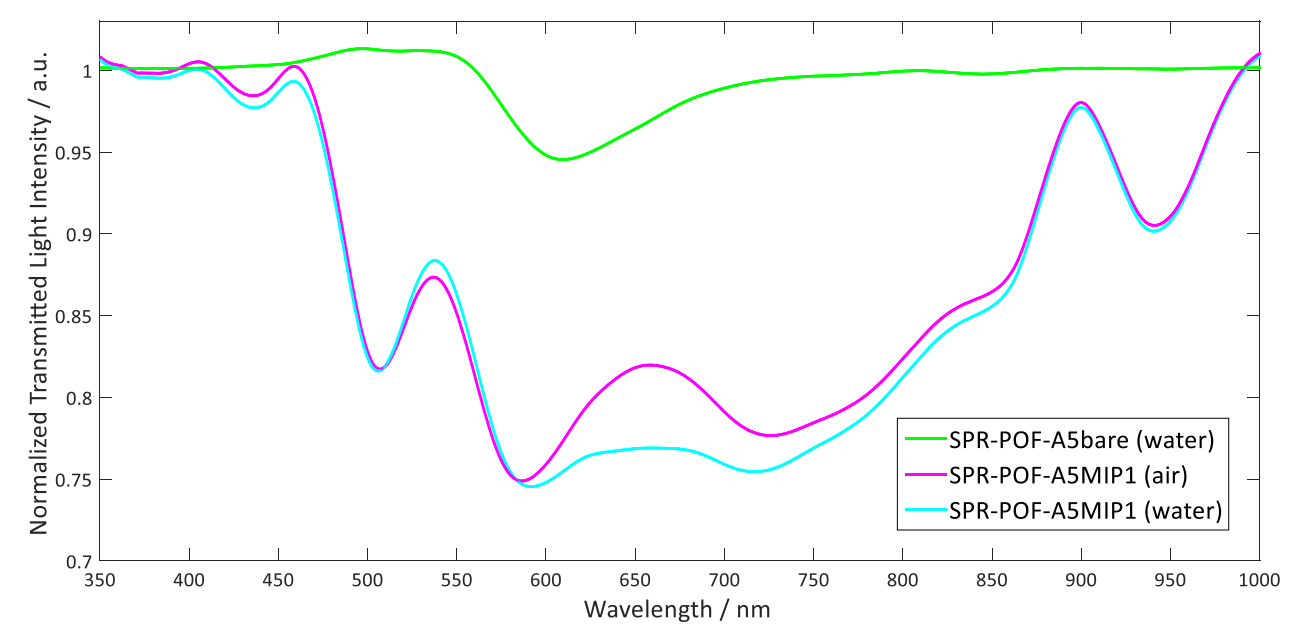

(a)

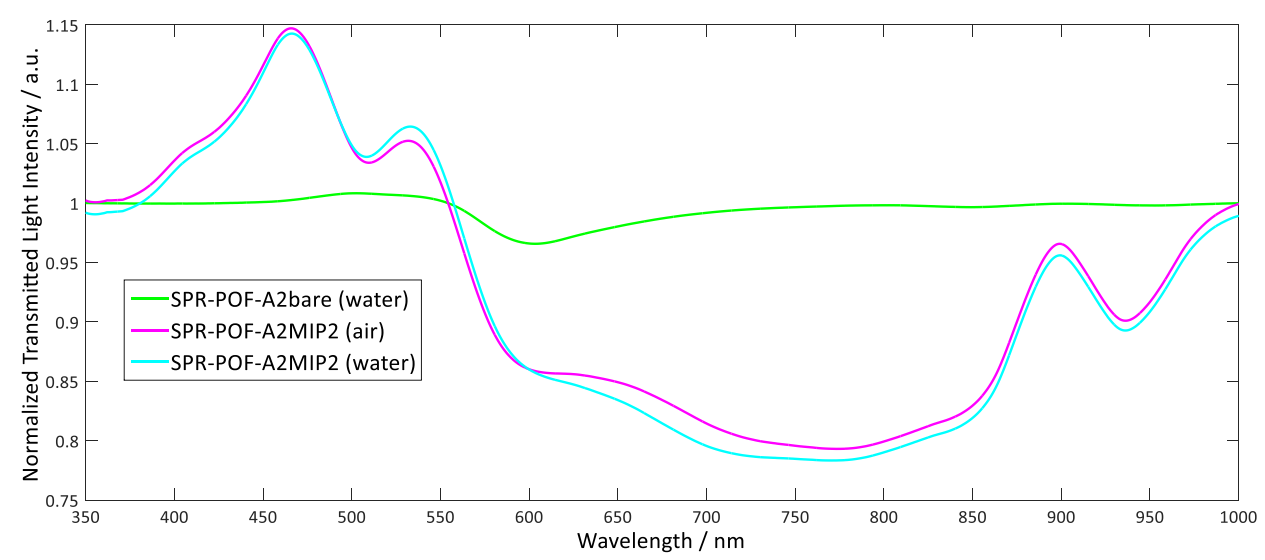

(b)

Figure 4. Spectra in air and water normalized on the spectrum of the corresponding bare sensor in air of the sensors: (a) SPR-POF-A5MIP1; (b) SPR-POF-A2MIP2. 
The effect of the different thicknesses can be more easily appreciated by comparing the same spectra but normalized to the SPR-POF-MIP in air, as reported in Figure 5. In both cases, the main resonance dip is shifted to higher wavelengths with respect to that obtained at the bare sensor, demonstrating that the MIP layer is present and that it modifies the RI of the dielectric layer. Its RI must be higher than that of water. As proposed in a previous work [14], the resonance wavelength shift with respect to that of the same platform not derivatized with MIP in pure water depends on the amount of MIP deposited, being different for SPR-POF-A5MIP1 and SPR-POF-A1MIP2.

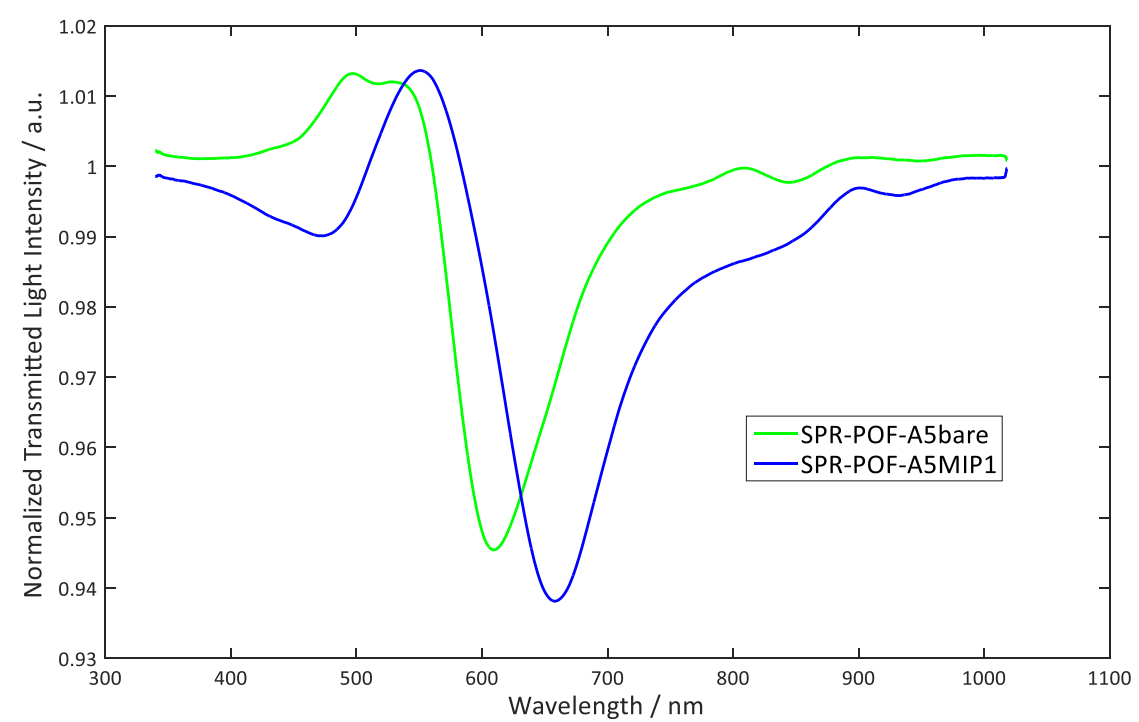

(a)

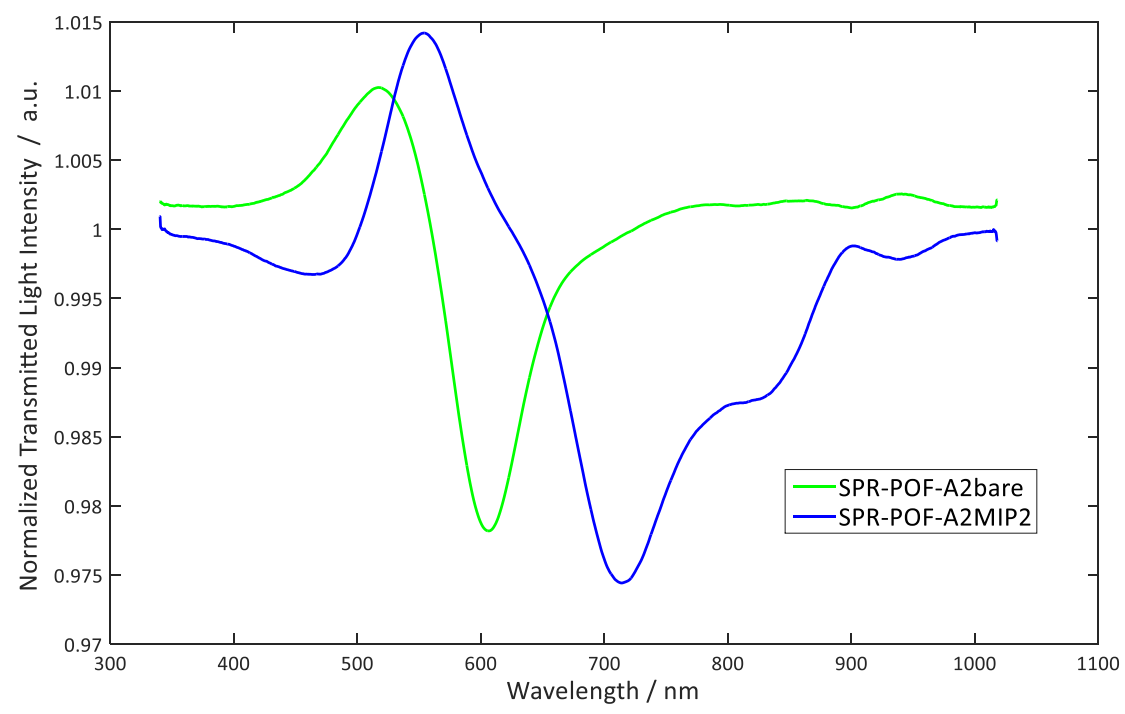

(b)

Figure 5. Spectra in water of the bare and MIP derivatized platforms normalized to the corresponding platform in air: (a) spectra of SPR-POF-A5bare and SPR-POF-A5MIP1 in water; (b) spectra of SPRPOF-A2bare and SPR-POF-A2MIP2.

By increasing the number of MIP layers, the resonance wavelength increases, and a shift to higher wavelengths (red shift) is observed (from $660 \mathrm{~nm}$ to $720 \mathrm{~nm}$ ). This could be due either to a direct sensing of the RI of the fluid overlaying the MIP or to some solvation effects. As a matter of fact, the polymer considered here contains carboxylic groups from the functional monomer MAA, which can easily interact with water. 


\subsection{SPR-POF-MIP EGDMA Sensor}

The MIP based on DVB as the cross-linker is highly lipophilic, which can be of help when dealing with oil samples, since a good contact between the receptor and the dissolved molecule is assured, but at the same time, the measurement in a drop is more difficult due to the fact that the drop tends to expand and fall. In order to reduce the lipophilic character of the MIP, a more polar cross-linker was used for the polymer preparation, EGDMA. Despite this, the surface of the sensor obtained was still lipophilic, since the oil drop expanded in the same way as that on the MIP DVB surface. The spectra of this sensor in different media normalized on the corresponding bare platform in air are reported in Figure 6.

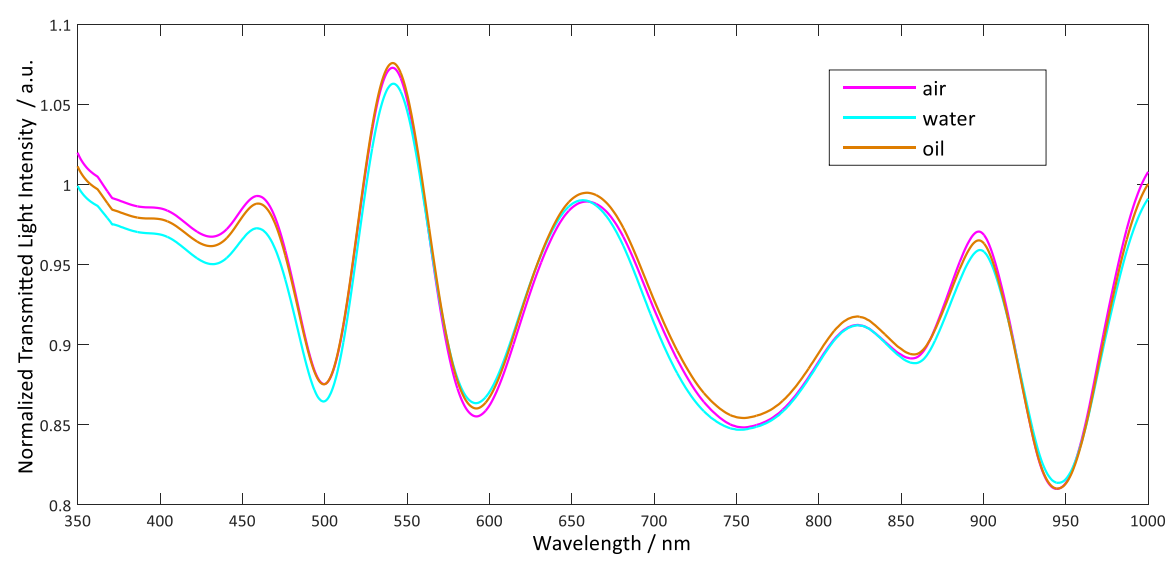

(a)

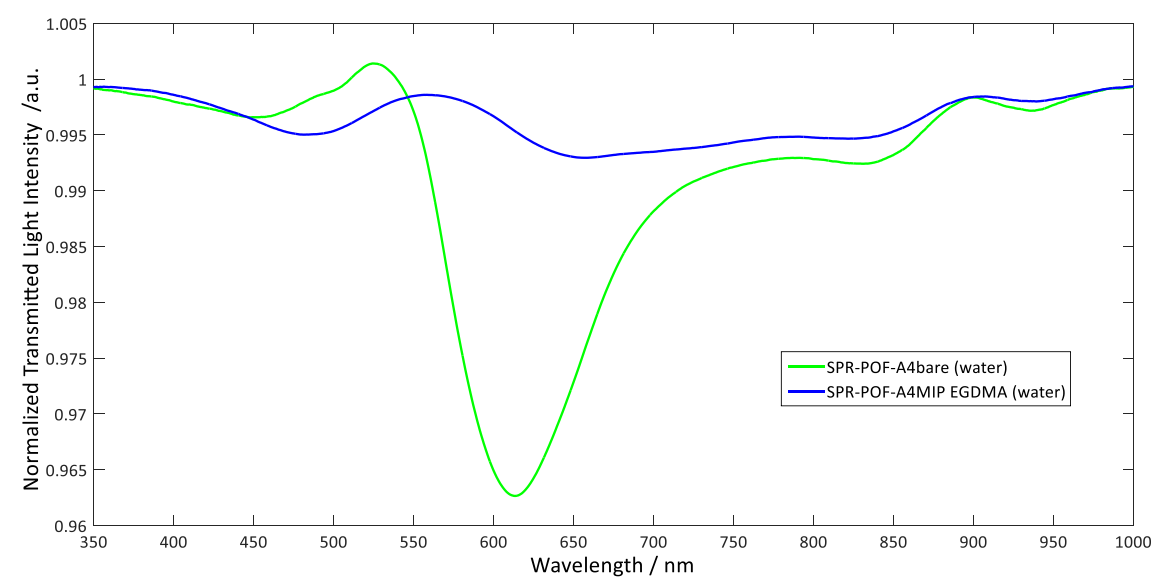

(b)

Figure 6. Spectra of SPR-POF-A4MIP EGDMA in different media: (a) normalized on the bare platform in air; (b) normalized on the MIP derivatized platform in air.

Spectra in air and water are very similar and so are those in oil, except that at about $740 \mathrm{~nm}$, which has a different shape. Moreover, the spectra are similar to those of the sensors with DVB-based MIP obtained by spinning the prepolymeric mixture at $1000 \mathrm{rpm}$, with well-defined multiple dips instead of a large band with multiple minima. The spectra in water normalized on the corresponding MIP EGDMA derivatized sensor in air are noticeably different from those obtained on SPR-POF-MIP sensors, since only shallow dips at $480 \mathrm{~nm}$ and $655 \mathrm{~nm}$ are formed. This indicates that the different cross-linker (EGDMA) produces a polymer that is practically insensitive to water, despite the polar nature of the cross-linker, probably because of the high rigidity. 


\subsection{Characterization of the Response of the SPR-POF-MIP Sensors}

The response of the sensor to the concentration of the substance of interest, 2-FAL, was investigated by determining the dose-response curve, and by applying the Langmuir adsorption model, assuming that the adsorption takes place through combination at specific sites, which in this case, are the imprinted sites. The model makes it possible to evaluate the affinity constant of the template for the MIP exactly in the form in which it is used in the sensing system, i.e., as a layer. This approach has been previously applied for the adsorption from water [14].

As an example, in Figure 7, the SPR spectra in oil, at different concentrations of 2-FAL, of the two sensors with MIP layers of different thickness, SPR-POF-A1MIP2 and SPR-POFA5MIP1, normalized on the corresponding bare sensor in air, are reported. The washing between successive standards consisted of five repeated flushing with ethanol.

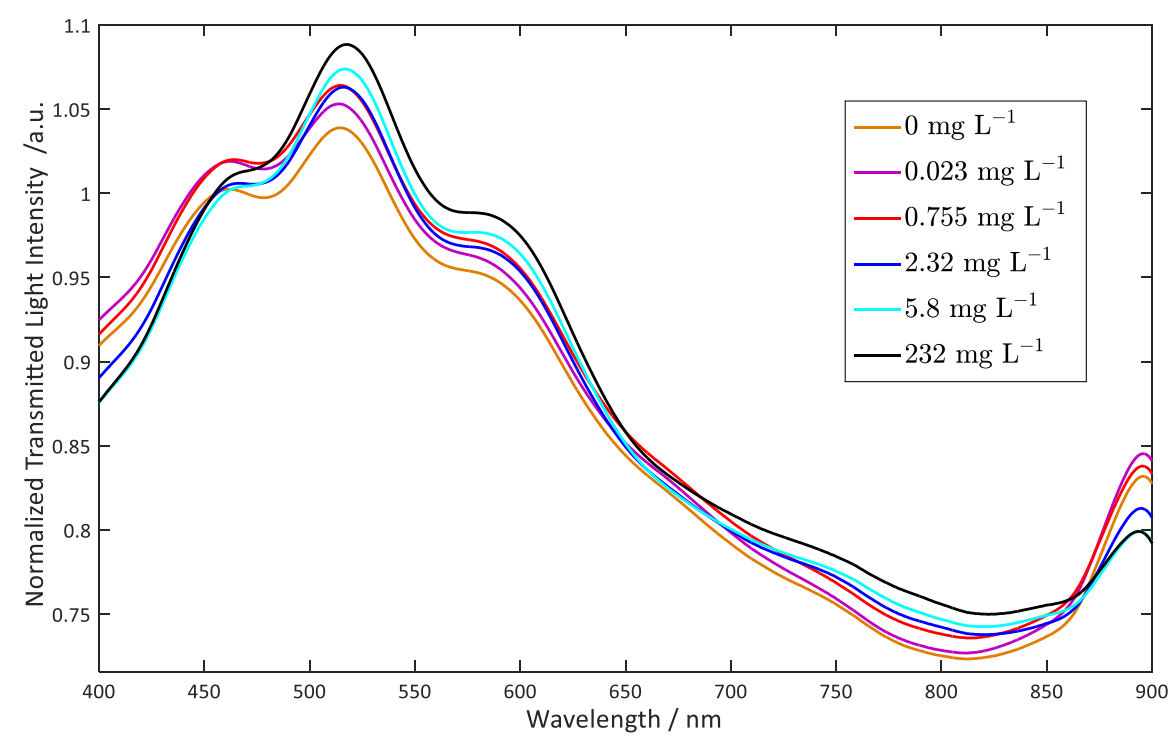

(a)

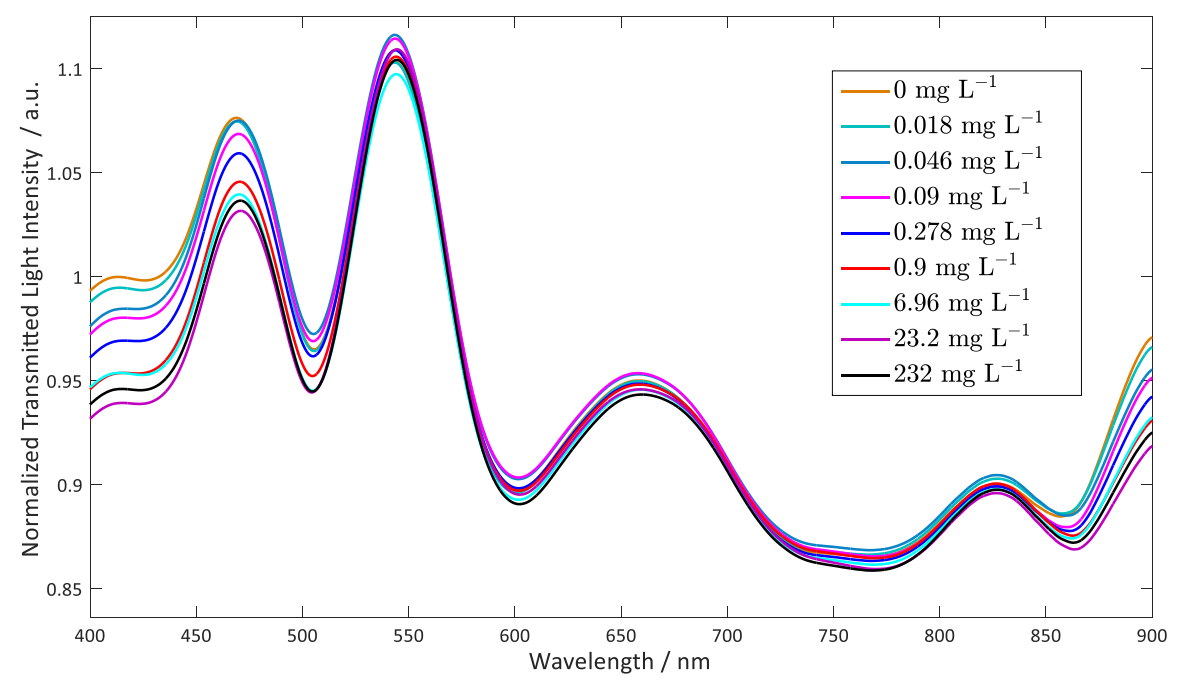

(b)

Figure 7. SPR spectra in oil, at different concentrations of 2-FAL, of two sensors with MIP layers of different thickness normalized on the corresponding bare sensor in air: (a) SPR-POF-A1MIP2; (b) SPR-POF-A5MIP1. 
In both cases, multiple resonance dips are present, much better defined in the case of the sensor with a lower amount of MIP deposited. The resonance depending on the 2-FAL concentration is, respectively, $880 \mathrm{~nm}$ and $812 \mathrm{~nm}$ for the two considered sensors. This indicates that in the case of SPR-POF-A1MIP2, the MIP layer is sufficiently thick to avoid the effect of the fluid over the MIP (no effect of the sample bulk), while in the case of SPR-POF-A5MIP1, which contains a lower amount of MIP, there is a strong effect of the RI of the overlying liquid, which is high for the considered oil, 1.47. Nevertheless the resonance wavelength is still useful, being $880 \mathrm{~nm}$.

The corresponding two standardization curves are shown in Figure 8.

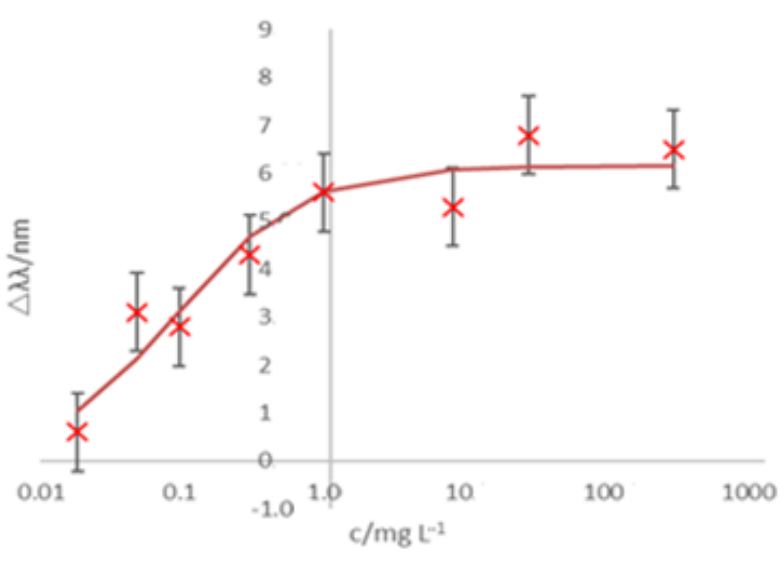

(a)

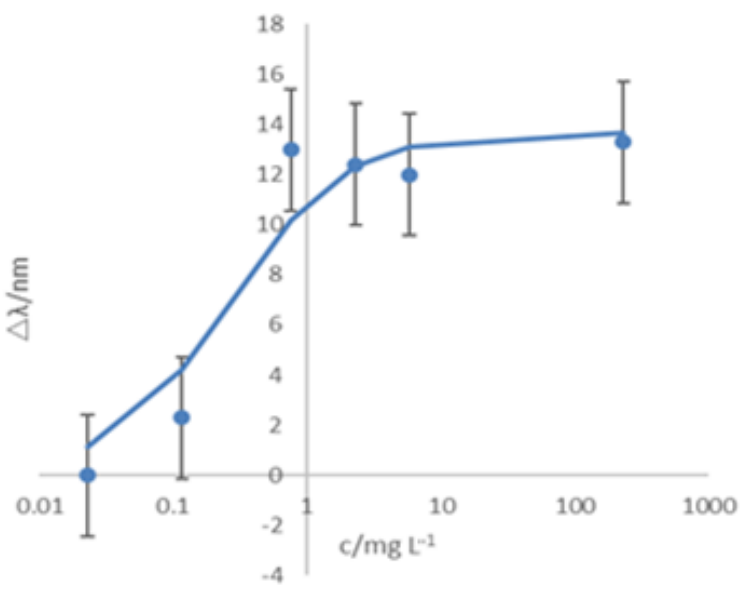

(b)

Figure 8. Dose-response curves for 2-FAL in vegetable oil of: (a) SPR-POF-A5MIP1; (b) SPR-POF-A1MIP2.

The dose-response curves are similar, but the maximum signal is higher when a higher amount of polymer is deposited, possibly corresponding to the presence of a larger number of accessible combination sites when more polymer is deposited. The parameters of the dose-response curve (Equation (1)) are reported in Table 1.

Table 1. Parameters of the dose-response curve for 2-FAL in vegetable oil for different SPR-POF-MIP sensors.

\begin{tabular}{|c|c|c|c|c|}
\hline & $K_{\mathrm{aff}}\left(\mathrm{L} \mathrm{M}^{-1}\right)$ & Sens $\left(\mathrm{nm} \mathrm{mg}^{-1} \mathrm{~L}\right)$ & SEy (nm) & $\operatorname{LOD}\left(\mu \mathrm{g} \mathrm{L}^{-1}\right)$ \\
\hline SPR-POF-A5MIP1 (N = 6) & $1.09 \times 10^{6}$ & 70 & 0.66 & 18 \\
\hline SPR-POF-A1MIP2 $(\mathrm{N}=5)$ & $3.69 \times 10^{5}$ & 53 & 1.69 & 64 \\
\hline SPR-POF-A4MIP EGDMA (N = 7) & $2.60 \times 10^{5}$ & 70 & 0.14 & 52 \\
\hline
\end{tabular}

SEy is the error of the response, $\Delta \lambda ; \mathrm{N}$ indicates the number of standards considered.

The error on the resonance wavelength shift, and the detection limit, is lower for SPR-POF-A5MIP1, for which the resonance dips are better defined, than for SPR-POFA1MIP2. The affinity is high enough to make the determination at a low concentration level possible, useful for application in different fields, for example for the determination of 2-FAL in vegetable oils. Nevertheless, it could be too high for other applications. A possible improvement could take place by applying a more extensive washing procedure.

For the sensor SPR-POF-A4MIP EGDMA, the spectra in oil, normalized to those of the bare platform in air, are similar to the spectra in water and in air. The peak at $850 \mathrm{~nm}$ is shifted to higher wavelengths when the concentration of 2-FAL increases. By applying the Langmuir model to the dose-response curve (Equation (1)), the parameters could be evaluated, and they are reported for comparison in Table 2. The affinity constant is lower for SPR-POF-A4MIP EGDMA than for the sensors prepared with DVD as the crosslinking agent, despite the fact that the functional polymer is always MAA at the same concentration. It could be guessed that some interaction between the MAA and EGDMA 
can somewhat lower the affinity of the sites for the template. Notice the lower value of SEy, which corresponds to the better sharpness of the peak obtained in this case.

Table 2. Parameters of the dose-response curve for 2-FAL in vegetable oil, at an SPR platform extensively cleaned, for different washing procedures between measurements.

\begin{tabular}{|c|c|c|c|c|c|}
\hline & $K_{\mathrm{aff}}\left(\mathrm{L} \mathrm{M}^{-1}\right)$ & Sens $\left(\mathrm{nm} \mathrm{mg} \mathrm{m}^{-1} \mathrm{~L}\right)$ & SEy (nm) & $\Delta \lambda_{\max }(\mathrm{nm})$ & $\operatorname{LOD}\left(\mu \mathrm{g} \mathrm{L}^{-1}\right)$ \\
\hline $\begin{array}{l}\text { SPR-POF-A2MIP2 }(\mathrm{N}=6) \text { stand } \\
\text { with washing in water }\end{array}$ & $8.32 \times 10^{7}$ & $8.18 \times 10^{7}$ & 0.13 & 0.98 & 0.3 \\
\hline $\begin{array}{c}\text { SPR-POF-A2MIP2 }(\mathrm{N}=5) \text { stand } \\
\text { with washing in ethanol }\end{array}$ & $1.77 \times 10^{8}$ & $2.70 \times 10^{8}$ & 0.19 & 1.52 & 0.14 \\
\hline
\end{tabular}

SEy is the error of the response, $\Delta \lambda ; \mathrm{N}$ indicates the number of standards considered.

The repeatability of the sensor was demonstrated by repeating three times every ten days (the sensor was stored in the air between the successive measurements) the doseresponse curve for 2-FAL in vegetable oil and evaluating the parameters of the curve. The average values with the relative standard deviation are: $K_{\text {aff }}=1.02(0.01) \times 10^{6} \mathrm{M}^{-1}$ and $\Delta \lambda_{\max }=8.2(2.9) \mathrm{nm}$. The repeatability, measured by the relative standard deviation, of the affinity constant is good, while that of $\Delta \lambda_{\max }$ is somewhat lower, which could be due to the poor reproducibility of the cleaning procedure.

\subsection{Effect of Extended Washing and Conditioning}

A sensor (SPR-POF-A2MIP2) was washed by flushing five times with ethanol, which is the usual procedure, and then further washed alternately with ethanol and the same oil used for the successive measurements. The idea is that while ethanol is able to extract 2-FAL and other hydrophilic components of the polymer, oil can extract hydrophobic impurities, which could cause a large irreproducibility during the successive measurements in oil if not completely extracted. This could also contribute to making stronger combination sites free. The spectra in oil with different concentrations of 2-FAL were then registered. The washing procedure of the sensor between successive standards consisted of five repeated flushing with ethanol. All the spectra were normalized on the same spectrum of the bare sensor in air. An example of the spectrum of a sensor with thicker MIP film in oil after extended extraction and by washing with ethanol between successive standards is reported in Figure 9.

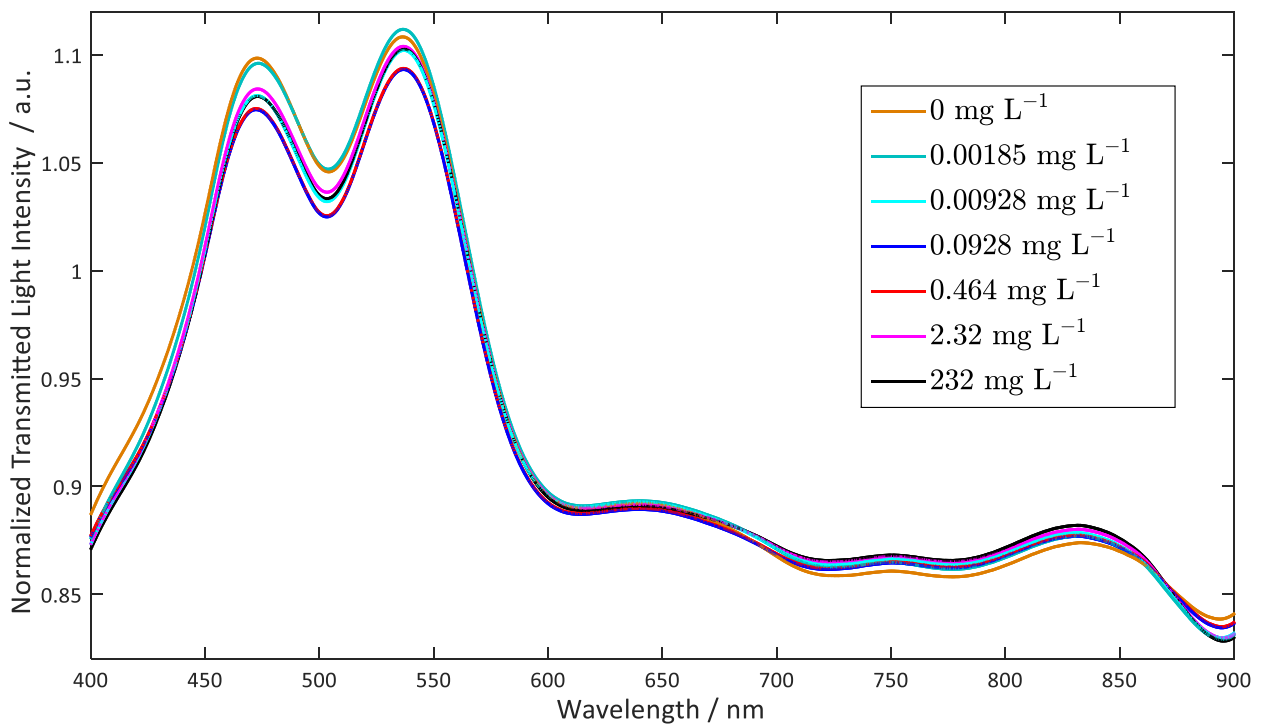

Figure 9. Spectra of SPR-POF-A2MIP2 (with extended cleaning) in oil. 
Notice the presence of the peak at $500 \mathrm{~nm}$, with two counter dips at $460 \mathrm{~nm}$ and $530 \mathrm{~nm}$, which could be due to the particular spectrum used for normalization, the same for all the reported curves.

Table 2 shows the parameters obtained from two dose-response curves, one obtained by washing in ethanol between successive standards and the other with water. The results are substantially similar.

The more relevant point is that the affinity constant of the sites revealed after extended washing is about two magnitude orders higher than that with rapid flushing with ethanol. As a consequence, the LOD was also much lower.

Evidently, only by this washing procedure, the 2-FAL molecules linked to the stronger interaction sites can be extracted.

\section{Discussion and Conclusions}

The spectra of the SPR-POF-MIP platforms in fluids with different refractive indices, such as air, water and oil, are characterized by the presence of multiple dips and even large bands, the general shape of which depends in part on the amount of polymer overlaying the gold resonant surface. Better-defined dips are obtained by the deposition of lower amounts of MIP; however, in this case, there is a larger effect of the RI of the overlaying medium, which is higher when the polymer layer is thinner. As a matter of fact, when dealing with samples with high RI, there is the possibility that the resonance wavelength exceeds the range of the detectable wavelength. However, it has been shown that this is not the case in the systems considered here, even when relatively thin MIP layers are deposited. The fact that in this kind of sensor better dips are obtained highly facilitates the evaluation of the peak position, i.e., the resonance wavelength. The thickness of the layer seems to have only a minor influence on the affinity of the MIP for 2-FAL and thus on the detection limit of the sensing device. An affinity constant of about $10^{6} \mathrm{M}^{-1} \mathrm{~L}$ is evaluated in the vegetable oil, near to that from water previously evaluated [14]. A large effect on the affinity is due to the washing procedure. An affinity constant about two orders of magnitude higher is found after extended cleaning, indicating that sites with higher affinity are made free.

Author Contributions: Conceptualization, M.P., N.C. and L.D.M.; Data curation, M.P. and L.D.M.; Formal analysis, M.P., N.C., L.Z. and L.D.M.; Investigation, M.P. and L.D.M.; Methodology, M.P., N.C., L.Z. and L.D.M.; Resources, M.P. and L.D.M.; Supervision, M.P., N.C. and L.D.M.; Validation, M.P. and L.D.M.; Writing—original draft, M.P., N.C., L.Z. and L.D.M.; Writing—review and editing, M.P., N.C. and L.D.M. All authors have read and agreed to the published version of the manuscript.

Funding: This research was funded by the Research Fund for the Italian Electrical System in compliance with the Decree of Minister of Economic Development 16 April 2018.

Institutional Review Board Statement: Not applicable.

Informed Consent Statement: Not applicable.

Data Availability Statement: The data are available on reasonable request from the corresponding author.

Acknowledgments: Authors acknowlege F. Scatiggio and G. Campi from A\&A Fratelli Parodi S.p.A for providing the vegetable oil samples.

Conflicts of Interest: The authors declare no conflict of interest.

\section{References}

1. Hoffmann, M.W.; Wildermuth, S.S.; Gitzel, R.; Boyaci, A.; Gebhardt, J.; Kaul, H.; Amihai, I.; Forg, B.; Suriyah, M.M. Integration of novel sensors and machine learning for predictive maintenance in medium voltage switchgear to enable the energy and mobility revolutions. Sensors 2020, 20, 2099. [CrossRef] [PubMed]

2. Lopez, P.; Mabe, J.; Miró, G.; Etxeberria, L. Low cost photonic sensor for in-line oil quality monitoring: Methodological development process towards uncertainty mitigation. Sensors 2018, 18, 2015. [CrossRef] [PubMed]

3. Lundgaard, L.E.; Hansen, W.; Linhjell, D.; Painter, T.J. Aging of oil-impregnated paper in power transformers. IEEE Trans. Power Deliv. 2004, 19, 230-239. [CrossRef] 
4. N'cho, J.S.; Fofana, I.; Hadjadj, Y.; Beroual, A. Review of physicochemical-based diagnostic techniques for assessing insulation condition in aged transformers. Energies 2016, 9, 367. [CrossRef]

5. N'cho, J.S.; Fofana, I. Review of fiber optic based diagnostic techniques used in power transformers. Energies 2020, 13, 1789. [CrossRef]

6. Leung, A.; Shankar, P.M.; Mutharasan, R. A review of fiber-optic biosensors. Sens. Actuators B Chem. 2007, 125, 688-703. [CrossRef]

7. Gupta, B.D.; Verma, R.K. Surface plasmon resonance-based fiber optic sensors: Principle, probe designs, and some applications. J. Sens. 2009, 2009, 1-12. [CrossRef]

8. Caucheteur, C.; Guo, T.; Albert, J. Review of plasmonic fiber optic biochemical sensors: Improving the limit of detection Anal. Bioanal. Chem. 2015, 407, 3883-3897. [CrossRef]

9. Wang, X.D.; Wolfbeis, O.S. Fiber-optic chemical sensors and biosensors (2008-2012). Anal. Chem. 2013, 85, 487-508. [CrossRef]

10. Wang, X.D.; Wolfbeis, O.S. Fiber-optic chemical sensors and biosensors (2013-2015). Anal. Chem. 2016, 88, 203-227. [CrossRef] [PubMed]

11. Cennamo, N.; Pesavento, M.; Zeni, L. A review on simple and highly sensitive plastic optical fiber probes for bio-chemical sensing. Sens. Actuators B Chem. 2021, 331, 129393. [CrossRef]

12. Cennamo, N.; D'Agostino, G.; Galatus, R.; Bibbò, L.; Pesavento, M.; Zeni, L. Sensors based on surface plasmon resonance in a plastic optical fiber for the detection of trinitrotoluene. Sens. Actuators B Chem. 2013, 188, 221-226. [CrossRef]

13. Cennamo, N.; D'Agostino, G.; Pesavento, M.; Zeni, L. High selectivity and sensitivity sensor based on MIP and SPR in tapered plastic optical fibers for the detection of L-nicotine. Sens. Actuators B Chem. 2014, 191, 529-536. [CrossRef]

14. Pesavento, M.; Zeni, L.; De Maria, L.; Alberti, G.; Cennamo, N. SPR-optical fiber-molecularly imprinted polymer sensor for the detection of furfural in wine. Biosensors 2021, 11, 72. [CrossRef] [PubMed]

15. Cennamo, N.; De Maria, L.; Chemelli, C.; Profumo, A.; Zeni, L.; Pesavento, M. Markers detection in transformer oil by plasmonic chemical sensor system based on POF and MIPs. IEEE Sens. J. 2016, 16, 7663-7670. [CrossRef]

16. Trouillet, A.; Ronot-Trioli, C.; Veillas, C.; Gagnaire, H. Chemical sensing by surface plasmon resonance in a multimode optical fibre. Pure Applied Opt. 1996, 5, 227-237. [CrossRef]

17. Cennamo, N.; Massarotti, D.; Conte, L.; Zeni, L. Low cost sensors based on SPR in a plastic optical fiber for biosensor implementation. Sensors 2011, 11, 11752-11760. [CrossRef] [PubMed]

18. Arshady, R.; Mosbach, K. Synthesis of substrate-selective polymers by host-guest polymerization. Makromol. Chem. 1981, 182, 687-692. [CrossRef]

19. Uzun, L.; Turner, A.P.F. Molecularly-imprinted polymer sensors: Realising their potential. Biosens. Bioelectron. 2016, 76, 131-144. [CrossRef] [PubMed]

20. Chen, L.; Wang, X.; Lu, W.; Wu, X.; Li, J. Molecular imprinting: Perspectives and applications. Chem. Soc. Rev. 2016, 45, $2137-2211$. [CrossRef] [PubMed] 